\title{
A new inhibitor of glucose-6-phosphate dehydrogenase blocks pentose phosphate pathway and suppresses malignant proliferation and metastasis in vivo
}

\author{
Luigi Mele ${ }^{1}$, Francesca Paino ${ }^{1}$, Federica Papaccio ${ }^{2}$, Tarik Regad $\mathbb{0}^{3}$, David Boocock $\mathbb{0}^{3}$, Paola Stiuso ${ }^{4}$, Angela Lombardi ${ }^{4}$, \\ Davide Liccardo ${ }^{1}$, Gabriella Aquino ${ }^{5}$, Antonio Barbieri ${ }^{6}$, Claudio Arra ${ }^{6}$, Clare Coveney ${ }^{3}$, Marcella La Noce', \\ Gianpaolo Papaccio (10) ${ }^{1}$, Michele Caraglia (D) $^{4}$, Virginia Tirino ${ }^{1}$ and Vincenzo Desiderio (1)
}

\begin{abstract}
Pentose phosphate pathway (PPP) is a major glucose metabolism pathway, which has a fundamental role in cancer growth and metastasis. Even though PPP blockade has been pointed out as a very promising strategy against cancer, effective anti-PPP agents are not still available in the clinical setting. Here we demonstrate that the natural molecule polydatin inhibits glucose-6-phosphate dehydrogenase (G6PD), the key enzyme of PPP. Polydatin blocks G6PD causing accumulation of reactive oxygen species and strong increase of endoplasmic reticulum stress. These effects are followed by cell cycle block in S phase, an about $50 \%$ of apoptosis, and $60 \%$ inhibition of invasion in vitro. Accordingly, in an orthotopic metastatic model of tongue cancer, $100 \mathrm{mg} / \mathrm{kg}$ polydatin induced an about 30\% tumor size reduction with an about $80 \%$ inhibition of lymph node metastases and $50 \%$ reduction of lymph node size $(p<0.005)$. Polydatin is not toxic in animals up to a dose of $200 \mathrm{mg} / \mathrm{kg}$ and a phase II clinical trial shows that it is also well tolerated in humans (40 mg twice a day for 90 days). Thus, polydatin may be used as a reliable tool to limit human cancer growth and metastatic spread.
\end{abstract}

\section{Introduction}

The pentose phosphate pathway (PPP) has recently been shown to have a crucial role in cancer cell growth by providing both nucleotide precursors, needed for proliferation, and NADPH used for both intracellular ROS detoxification and catabolic metabolism ${ }^{1-5}$. The inhibition of PPP key enzymes, including glucose-6-phosphate dehydrogenase

Correspondence: Gianpaolo Papaccio (gianpaolo.papaccio@unicampania.it) or Michele Caraglia (michele.caraglia@unicampania.it)

${ }^{1}$ Department of Experimental Medicine, University of Campania "Luigi Vanvitelli", 80138 Naples, Italy

${ }^{2}$ Oncologia Medica ed Ematologia, Dipartimento Medico-Chirurgico di Internistica Clinica e Sperimentale "F. Magrassi e A. Lanzara", University of Campania "Luigi Vanvitelli", 80138 Naples, Italy

Full list of author information is available at the end of the article

These authors contributed equally: Luigi Mele, Francesca Paino, Federica Papaccio.

These authors jointly supervised this work: Michele Caraglia, Virginia Tirino, Vincenzo Desiderio.

Edited by G. Raschellã
(G6PD), strongly affects cancer cell proliferation in vitro, as well as in vivo ${ }^{6,7}$. G6PD is upregulated in many human cancers and correlates with poor prognosis ${ }^{8-12}$, whereas cancer patients harboring G6PD mutation show longer survival and reduced metastases ${ }^{13-15}$. Moreover, G6PD activity can be regulated by oncogenes such as PI3k/AKT, Ras, Src, mTORC1, or by oncosuppressors such as p53 and TAp73 ${ }^{4}$. Interestingly, the inhibition of G6PD may restore sensitivity of cancer cells to chemotherapy ${ }^{16}$. Therefore, PPP inhibition has been proposed as an attractive therapeutic strategy against cancer ${ }^{17}$. However, the inhibition of G6PD in clinical settings is hampered by the lack of specific inhibitors. To our knowledge, the only G6PD inhibitor ever used in vivo is the dehydropiandrosterone (DHEA),

\section{(c) The Author(s) 2018}

(c) (i) Open Access This article is licensed under a Creative Commons Attribution 4.0 International License, which permits use, sharing, adaptation, distribution and reproduction c. in any medium or format, as long as you give appropriate credit to the original author(s) and the source, provide a link to the Creative Commons license, and indicate if changes were made. The images or other third party material in this article are included in the article's Creative Commons license, unless indicated otherwise in a credit line to the material. If material is not included in the article's Creative Commons license and your intended use is not permitted by statutory regulation or exceeds the permitted use, you will need to obtain permission directly from the copyright holder. To view a copy of this license, visit http://creativecommons.org/licenses/by/4.0/. 
an endogenous steroid hormone, which is produced by adrenal glands acting as a metabolic precursor of androgen and estrogen. DHEA is rapidly converted into steroid hormones in vivo and its efficacy as an inhibitor of G6PD is under dispute ${ }^{18}$.

Polydatin (3,4',5-trihydroxystilbene-3- $\beta$-d-glucoside; transresveratrol $3-\beta$-mono-D-glucoside; piceid) is a natural molecule found in Polygonum cuspidatum and other plants. Polydatin is a glucoside of resveratrol and, together with other polyphenols, has been shown to have several biological effects, including the induction of apoptosis in carcinoma cells ${ }^{19-22}$. Here we have studied the effects of polydatin on G6PD activity, ROS levels, ER stress, and programmed cell death, and its role in inhibiting cancer cell proliferation and invasion both in vitro and in vivo.

\section{Results}

\section{Polydatin inhibits cancer cell proliferation and cell cycle progression}

We assessed the viability of head and neck squamous cell carcinoma (HNSCC) cell lines after polydatin treatments at different concentrations (from 2 to $100 \mu \mathrm{M}$ at 24 or $48 \mathrm{~h}$ ), by MTT assay. We found that polydatin-reduced cell viability in a dose- and time-dependent manner at an EC50 of $22 \mu \mathrm{M}$ for $24 \mathrm{~h}$ and $17 \mu \mathrm{M}$ for $48 \mathrm{~h}$, respectively (Fig. 1a). Based on these data we have selected, for all the subsequent experiments, concentrations of 10,20 , and 30 $\mu \mathrm{M}$ that represent the EC25, EC50, and EC75. To confirm the effects on cell viability, we performed an apoptosis assay based on Annexin V/PI staining (Fig. 1b and S1A). We observed a dose- and time-dependent reduction of cell viability and an increased apoptosis and necrosis of treated cells. Polydatin affect, as well the cell cycle inducing a block in the $S$ phase that reflected the ability of polydatin to inhibit cell proliferation (Fig. 1c and Fig. S1B). These results demonstrate that polydatin reduces viability, increases apoptosis, and prevents cell cycle progression of primary HNSCC cells. Similar results were obtained on breast cancer MCF7 cell line (Fig. S2D-F).

\section{Polydatin induces ER stress-driven cell death}

To understand the molecular mechanism leading to cell death, we hypothesized that polydatin could cause endoplasmic reticulum (ER) stress, which in turn can lead to apoptosis. We performed an immunofluorescence staining (IF) using the vital dye ER-Tracker, which accumulate into ER. As a positive control, we used tunicamycin, a known inducer of ER stress. Following treatment with either tunicamycin or different concentrations of polydatin, we observed an increased fluorescence, which corresponds to an enlarged ER (Fig. 1d). Inositol-requiring enzyme 1 (IRE1) is an enzyme with intrinsic kinase and endoribonuclease activity that is activated during ER stress. After oligomerization and auto-phosphorylation, IRE1 acts on X-box binding protein 1 (XBP1) mRNA and causes an unconventional alternative splicing that activates XBP1 transcription factor to upregulate ER chaperones. Clusters of oligomerization of phospho-IRE1 were observed in both polydatin and tunicamycin-treated cells by both IF (Fig. 1e) and immunoblotting (Fig. 1f). Moreover, a significant increase of XBP1 mRNA and its spliced form (sXBP1 mRNA) was observed. This effect was dose- and time-dependent and began $4 \mathrm{~h}$ following treatment (Fig. 1f). Following ER stress, both IRE1 and PERK (protein kinase RNA-like ER kinase) induce the transcription of CCAAT-enhancerbinding protein homologous protein (CHOP), which is involved in the activation of apoptosis. As expected, the phosphorylated form of PERK and two of its downstream effectors p-eIF2 $\alpha$ and ATF4 increased after polydatin treatment (Fig. 1g). Finally, CHOP transcript and protein also increased in response to polydatin treatment in a dose- and time-dependent manner as early as $2 \mathrm{~h}$ after treatment (Fig. 1f, g). ER chaperons such as BiP and CRT increased after treatment too, while CNX did not show significant changes upon treatment. To understand whether ER stress was driving cell death, we showed that the block of IRE1 or PERK activation, by specific inhibitors or by knockdown, partially reverted polydatin-induced cytotoxicity (Fig. 2a).

\section{Polydatin inhibits G6PD and induces oxidative and ER stresses}

To additionally investigate the molecular mechanism of action of polydatin, we performed a mass spectrometry protein expression analysis of cell lysates from untreated and polydatin-treated cells (Fig. S2A and S2B) that was compared with one from tunicamycin-treated cells. As expected, the expression of proteins involved in ER stress was affected by tunicamycin treatment (Table S1). Polydatin-treated cells also showed a pattern of expression that was consistent with stress response and which overlapped that one of tunicamycin-treated cells. Among the proteins modulated by polydatin, we found a group of proteins belonging to the family of oxidoreductases and that were not altered in tunicamycin-treated cells (Fig. S2B and Table S1). Specifically, we found that seven oxidoreductases, including isocitrate dehydrogenase 1 (IDH1), G6PD and 6-phosphogluconate-dehydrogenase (6PGD), were significantly upregulated in polydatin treated samples (24 h treatment). G6PD and 6PGD are both part of PPP and together with IDH1 are responsible for the production of almost all the cytosolic NADPH, which is necessary for redox balance. Indeed, we found a significant reactive oxygen species (ROS) accumulation following polydatin treatment (Fig. 2b, S5). ROS plays a critical role in many cellular processes and can be produced in the cytosol and in several organelles, including 


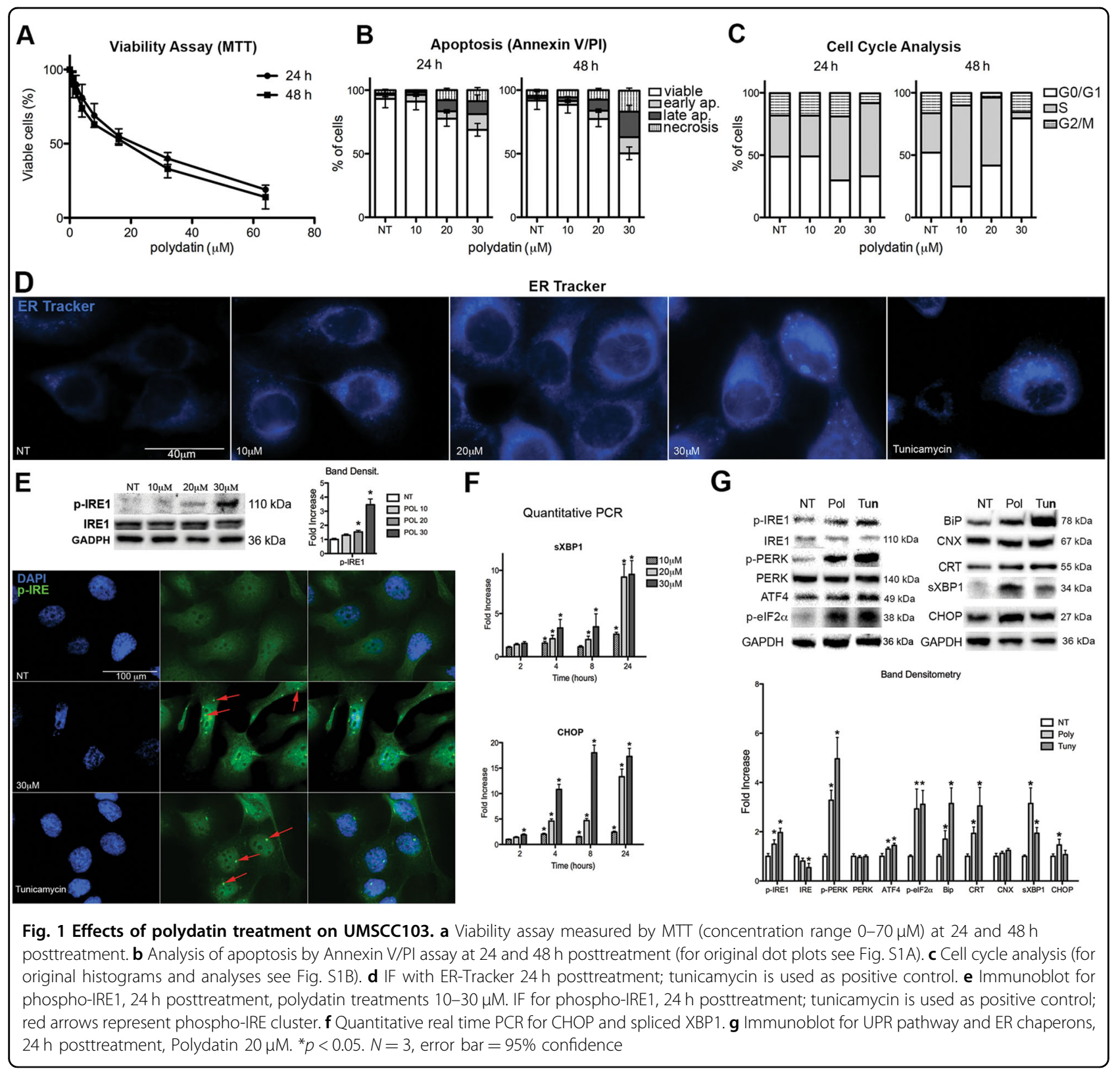

ER and mitochondria. To determine the role of ROS accumulation in ER stress, we incubate the cells with the antioxidant $N$-acetylcysteine (NAC), prior to polydatin treatment. Pre-treatments with NAC completely abrogated ER stress induced by polydatin, suggesting a causative link between oxidative and ER stress (Fig. 2c).

As G6PD is the PPP limiting enzyme, its inhibition would affect NADPH production, and results in redox imbalance. Thus, we hypothesized that the biological effects observed could be a consequence of G6PD block. Therefore, we performed a G6PD enzyme activity assay on carcinoma protein lysates, as well as on purified G6PD. In both cases, polydatin-inhibited G6PD activity in a concentration-dependent manner (Fig. 2d, S2C). This was paralleled by an increase of the NADP + /NADPH ratio in a time-dependent manner (Fig. 2e). These results suggest that polydatin inhibits G6PD causing an imbalance in NADP + /NADPH ratio that leads to an increase of oxidative stress. The capacity of tumor cells to control oxidative stress through NADPH production is directly correlated with their ability to migrate. Indeed, in vitro invasion assays confirmed that polydatin-inhibited cancer cell invasion in a dose-dependent manner (Fig. 2f).

\section{G6PD overexpression counteracts polydatin antitumor effects}

To confirm that G6PD inhibition was functionally related to the biological effects induced by polydatin, we 


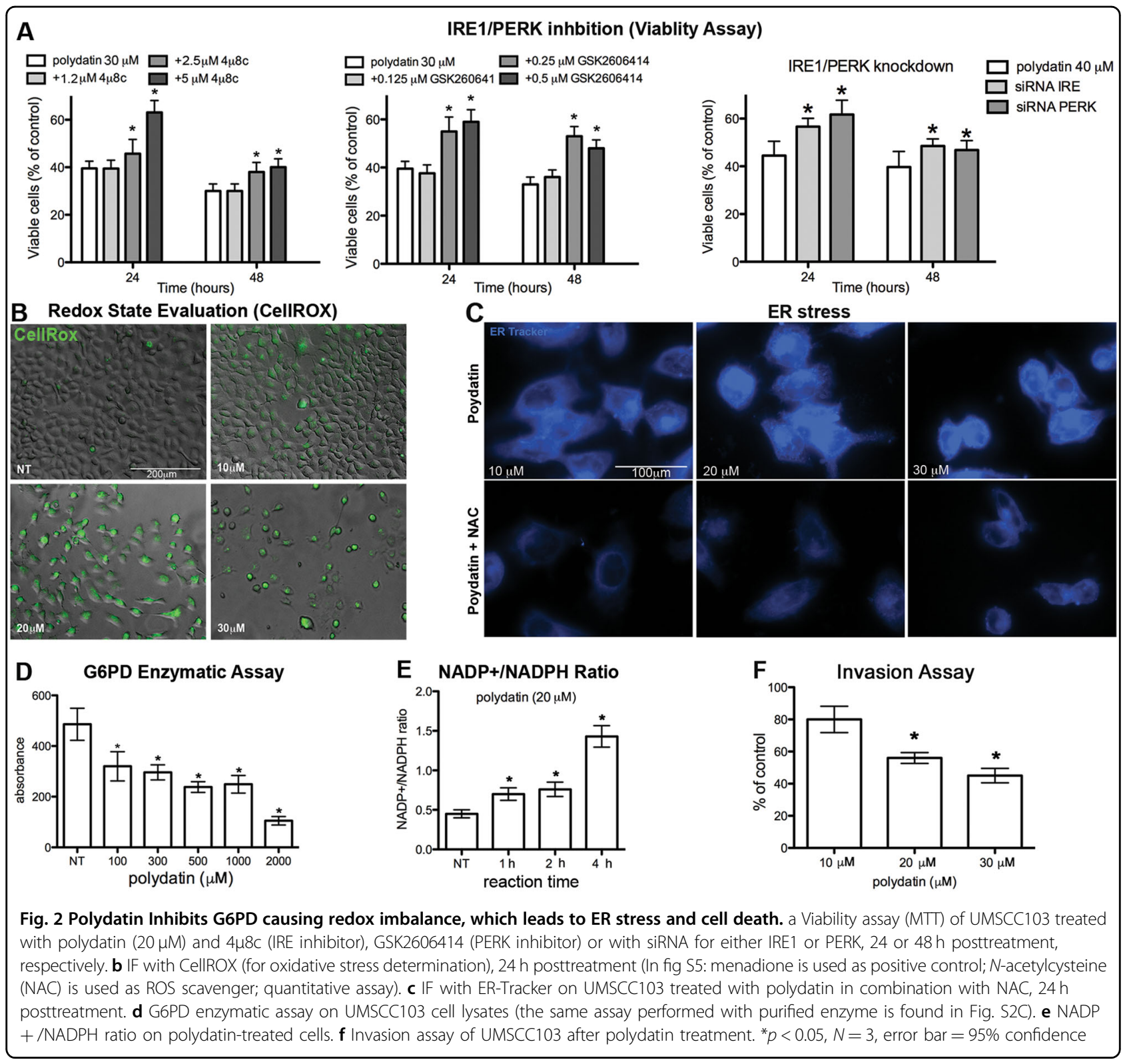

generated a G6PD overexpressing cell line $\left(\mathrm{MCF}^{\mathrm{G} 6 \mathrm{PD}+}\right)$ (Fig. S3A). MCF7 ${ }^{\mathrm{G} 6 \mathrm{PD}+}$ were resistant to polydatin treatment in both viability assay and apoptosis analyses. Indeed, $70 \mu \mathrm{M}$ polydatin at $24 \mathrm{~h}$ cause a $50 \%$ reduction in viability in mock cells vs $35 \%$ in $\mathrm{MCF}^{\mathrm{G} 6 \mathrm{PD}+}$. Moreover, $35 \mu \mathrm{M}$ polydatin at $48 \mathrm{~h}$ caused $65 \%$ mortality in mock vs $40 \%$ in $\mathrm{MCF}^{\mathrm{G} 6 \mathrm{PD}+}$ (Fig. S3B). Polydatin treatment caused a strong and significant increase of the $\mathrm{S}$ phase of cell cycle at $17.5 \mu \mathrm{M}(16 \pm 5 \%$ to $63 \pm 10 \%)$, while it increased both S and G1 phase at the expense of G2/M at 35 and $70 \mu \mathrm{M}$ in mock-transfected cells at $48 \mathrm{~h}$. Analysis of cell cycle at $48 \mathrm{~h}$ showed always an increase of $\mathrm{S}$ and $\mathrm{G} 2 / \mathrm{M}$ phases. On the other hand, the $\mathrm{MCF}^{\mathrm{G} 6 \mathrm{PD}+}$ cell cycle, was much less perturbed by polydatin that induced only a small increase of $\mathrm{S}$ phase at a concentration of 35 $\mu \mathrm{M}$ at $48 \mathrm{~h}$ (from $20 \pm 5 \%$ to $48 \pm 6 \%$ ) (Fig. S3C). $\mathrm{MCF}^{\mathrm{G} 6 \mathrm{PD}+}$ cells were resistant to ER stress induced by polydatin as showed by ER-Tracker staining (Fig. S3D). On the other hand, tunicamycin induced a strong ER stress in both mock and MCF7 ${ }^{\mathrm{G} 6 \mathrm{PD}+}$ cells. This result suggests that $\mathrm{MCF}^{\mathrm{G} 6 \mathrm{PD}+}$ are not resistant to ER stress induced by mechanisms independent from redox imbalance. To further confirm this, we show that $\mathrm{CHOP}$ and spliced-XBP1 transcripts were significantly higher in polydatin-treated mock cells than $\mathrm{MCF}^{\mathrm{G} 6 \mathrm{PD}+}$ (Fig. 3b). The phosphorylation and clustering of IRE1 was strong and evident in mock cells but completely undetectable in $\mathrm{MCF7}^{\mathrm{G} 6 \mathrm{PD}+}$ (Fig. S3D). Analysis of redox status after 


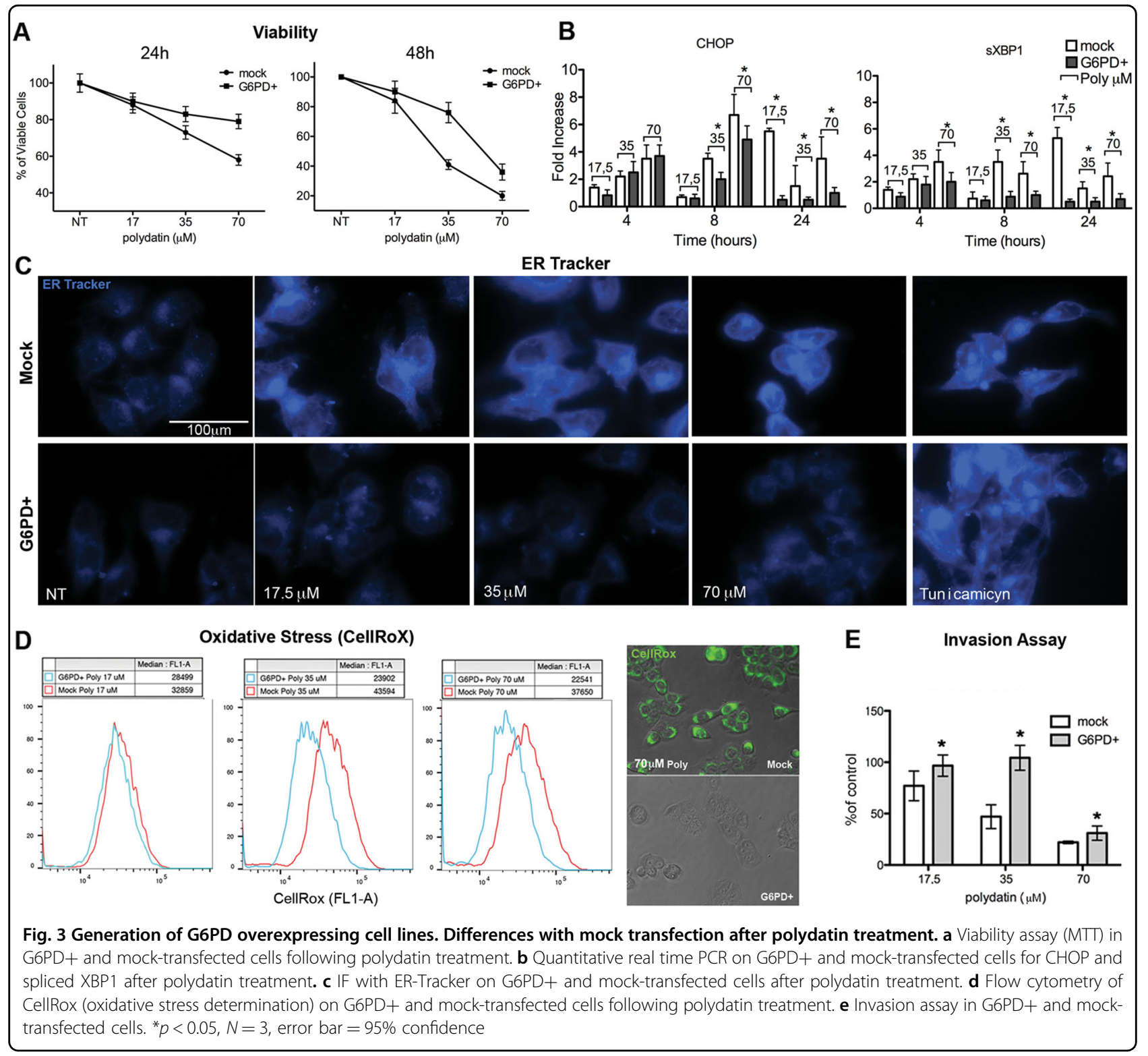

polydatin treatment revealed an about two-fold increase of ROS accumulation in mock cells at concentration above $17.5 \mu \mathrm{M}$ (Fig. S3D) but no effect in $\mathrm{MCF}^{\mathrm{G} 6 \mathrm{PD}+}$. Again, invasion ability of $\mathrm{MCF}^{\mathrm{G} 6 \mathrm{PD}+}$ was in the same way much less inhibited by polydatin treatment if compared to mock cells (Fig. S3E).

\section{Polydatin prevents lymph node metastases in an experimental orthotopic model of cancer}

Polydatin-induced cancer cell death and blocked invasion in vitro. Therefore, we developed an orthotopic metastatic model of oral cancer by injecting UMSCC103 in mice tongues, assessing the timing of tumor growth and lymph node metastases occurrence. Primary tumors were examined at the end of the experiment when mice were killed, while lymph node metastases were followed using ultrasound imaging. Primary tumor growth was determined by weighting entire tongues and showed statistically significant differences between polydatin-treated and -untreated mice (Fig. 4a). The reduction of primary tumors' growth was $\leq 30 \%$. The squamous cell carcinoma nature of the implanted tumor was confirmed by an expert pathologist (Fig. 4a). Ultrasound imaging and size determination of cervical lymph nodes revealed a much greater effect of polydatin on metastases (Fig. 4c, d). In details, the average size of untreated mice lymph nodes was $5 \pm 0.5 \mathrm{~mm}^{2}$ vs $3 \pm 0.3 \mathrm{~mm}^{2}(p<0.005)$ for the treated ones. These data were confirmed after mice killing and 


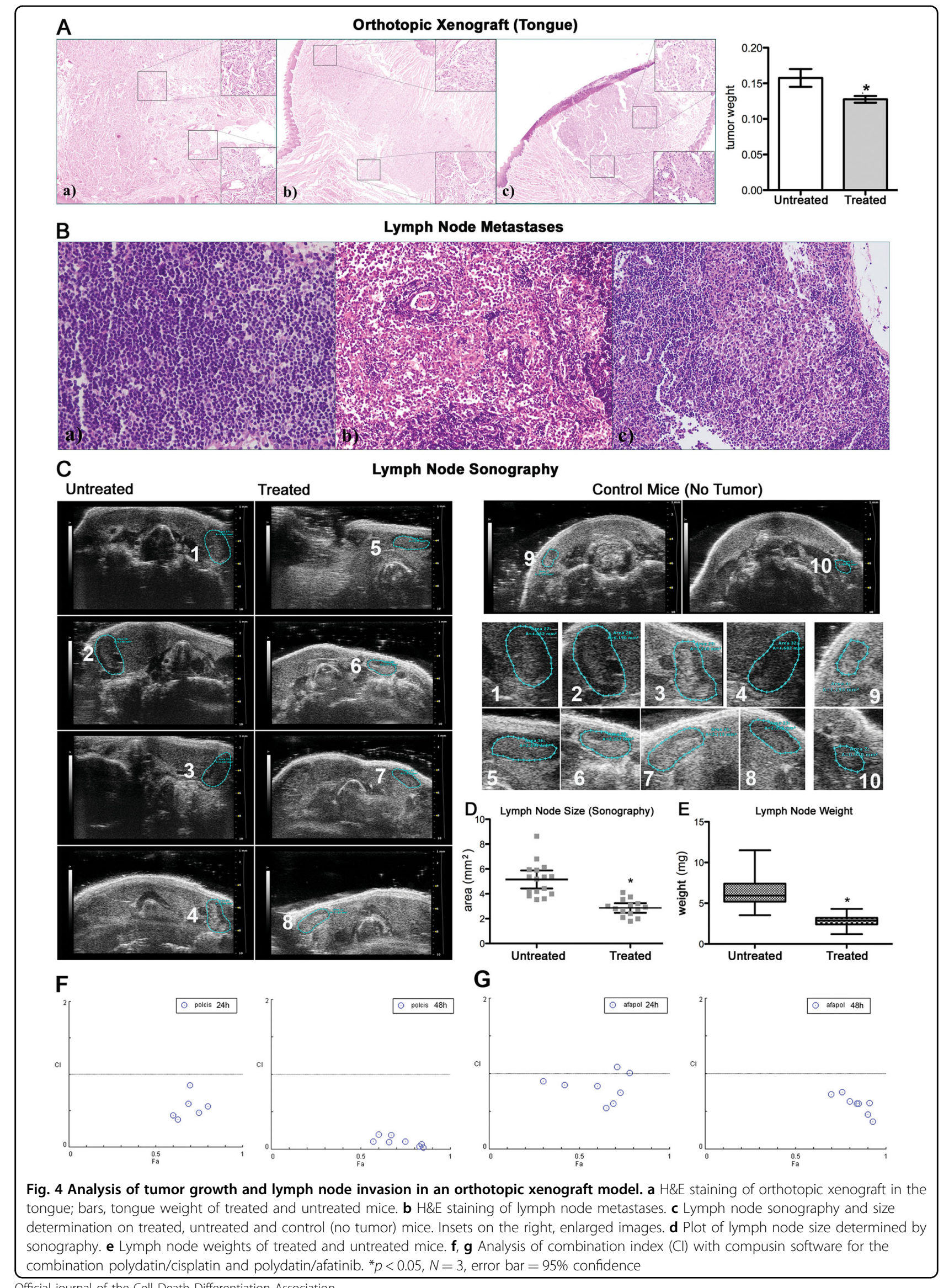

Official journal of the Cell Death Differentiation Association 
when lymph nodes were removed, weighted, and examined by a pathologist. We were able to identify and remove about $5 / 6$ cervical lymph nodes per mouse. Indeed, the average weight of untreated mice was $6.2 \pm$ $0.3 \mathrm{mg}$ vs $2.9 \pm 0.1 \mathrm{mg}$ for the treated ones $(p<0.005)$ (Fig. 4e). All lymph nodes were then examined for metastases and we found that only two of the treated mice (out of ten) had lymph node metastases, while eight out of ten were observed in untreated ones.

\section{G6PD inhibition increase the effect of chemotherapy}

PPP has been suggested to be involved in drug resistance in both solid tumors and leukemia. Two major work of Gregory et al. ${ }^{23}$ and Catanzaro et al. ${ }^{16}$ describe an involvement of G6PD in the resistance to cisplatin and tyrosine kinase inhibitor. For this reason, we performed analysis of synergism for the combination of cisplatin/polydatin and afatinib/polydatin. Both combinations were highly synergic at all the concentration used (Fig. 4f, g; S4).

\section{Discussion}

In the present manuscript, we have studied the effect of polydatin on cancer cells elucidating its biochemical mechanism of action and biological effects. Based upon our results, we provide evidence showing that polydatin induces a potent cancer cell growth inhibition paralleled by a strong reduction of invasive properties of cancer cells in vitro and in vivo. The latter effect was reported for the first time and represents a major goal of cancer research, as cancer metastases control is an unmet need in cancer treatment. We demonstrate that polydatin directly inhibits G6PD, the limiting enzyme of PPP, causing redox imbalance, which results in ER stress, cell cycle arrest, and apoptosis. To elucidate the molecular mechanism of polydatin, we performed a quantitative proteomic analysis of lysates from polydatin-treated cells at multiple time points, and compared the protein expression profiles with tunicamycin-treated cells. This approach enabled the identification of a group of enzymes that significantly increased following the treatment with polydatin but not with tunicamycin. The enzymes belonged to the family of oxidoreductases and among these, three enzymes drew our attention: IDH1, G6PD, and 6PGD. These three enzymes are involved in glucose metabolism and account for the production of most of the NADPH. The latter has been proposed as rate limiting for cell proliferation ${ }^{24-26}$. Moreover, NADPH has been demonstrated to be fundamental for cancer growth and metastases ${ }^{27}, 28$. Indeed, we found that polydatin inhibited G6PD in either a cell lysate or using the purified enzyme. To demonstrate that the cytotoxic effects of polydatin were mediated by G6PD inhibition, we generated a cell line overexpressing G6PD. In these cells, treatment with polydatin resulted in a much lower effect if compared to control cells. This was observed for all the biological parameters, we analyzed. G6PD inhibition blocks proliferation and reduces DNA synthesis $^{29}$, an effect similar to that one observed when cancer cells are treated with polydatin that inhibits cell cycle at the $S$ phase ${ }^{30}$. In addition, the accumulation of cells in S phase of cell cycle at the expenses of G2/M is consistent with nucleotides shortage ${ }^{31}$. It has to be highlighted that, although polydatin has been used for more than 30 years, the molecular mechanism responsible for the effects reported have never been elucidated. We showed, for the first time, that the biological effects produced by polydatin treatment depend on G6PD inhibition and PPP block. This is, in our opinion, a pivotal finding, as specific G6PD or PPP inhibitors are not currently available in clinical setting. Indeed, although the PPP has been identified as a target for cancer therapy $4,7,9,17,24$ and recently pointed out to be responsible for drug resistance in humans by a relevant study of Gregory et al. ${ }^{23}$, the lack of specific inhibitors hampers research and clinical application that aim to target this pathway. The widely used G6PD inhibitor DHEA is rapidly converted in vivo into other hormones, which makes this drug not active ${ }^{18}$. The inhibitory effect recorded in some clinical trials seems to be more luckily produced by the interaction between DHEA and estrogen receptors. Moreover, the results obtained with DHEA in vitro need to be interpreted very carefully due to potential biasing by the several biological effects of this hormone. Interestingly, the capacity of tumor cells to control oxidative stress through NADPH production is directly correlated with their ability to form metastases in vivo ${ }^{32}$. Moreover, Richardson et al. $^{33}$, used breast epithelial MCF10A cells (the parental and benign MCF10A, premalignant MCF10AT, and malignant MCF10CA1a) and showed that PPP flux increases with malignancy and correlates with tumor aggressiveness $^{33}$. Consistently, the PPP is associated with invasiveness and seems to play a crucial role during the metastatic process by protecting metastatic cells ${ }^{5}, 34$. Therefore, our findings demonstrate that polydatin inhibits cancer cell invasion through PPP-dependent NADPH decrease. On the bases of these findings, we developed an orthotopic and metastatic model of oral cancer showing that polydatin strongly reduced both tumor growth and lymph node metastases. Interestingly, polydatin has previously been administered in different animal models to a dose up to $200 \mathrm{mg} / \mathrm{kg}$ with no reported major cardiovascular, hepatic, bone marrow, and renal toxicities ${ }^{35-37}$. Pharmacokinetic studies showed that polydatin is absorbed and distributed to tissues if given intravenously or by oral administration ${ }^{38}$. Phase II clinical trials have been performed using polydatin at $20-40 \mathrm{mg}$ twice a day for a period, as long as 3 months and none of those reported any major cardiovascular, hepatic, bone marrow, and 


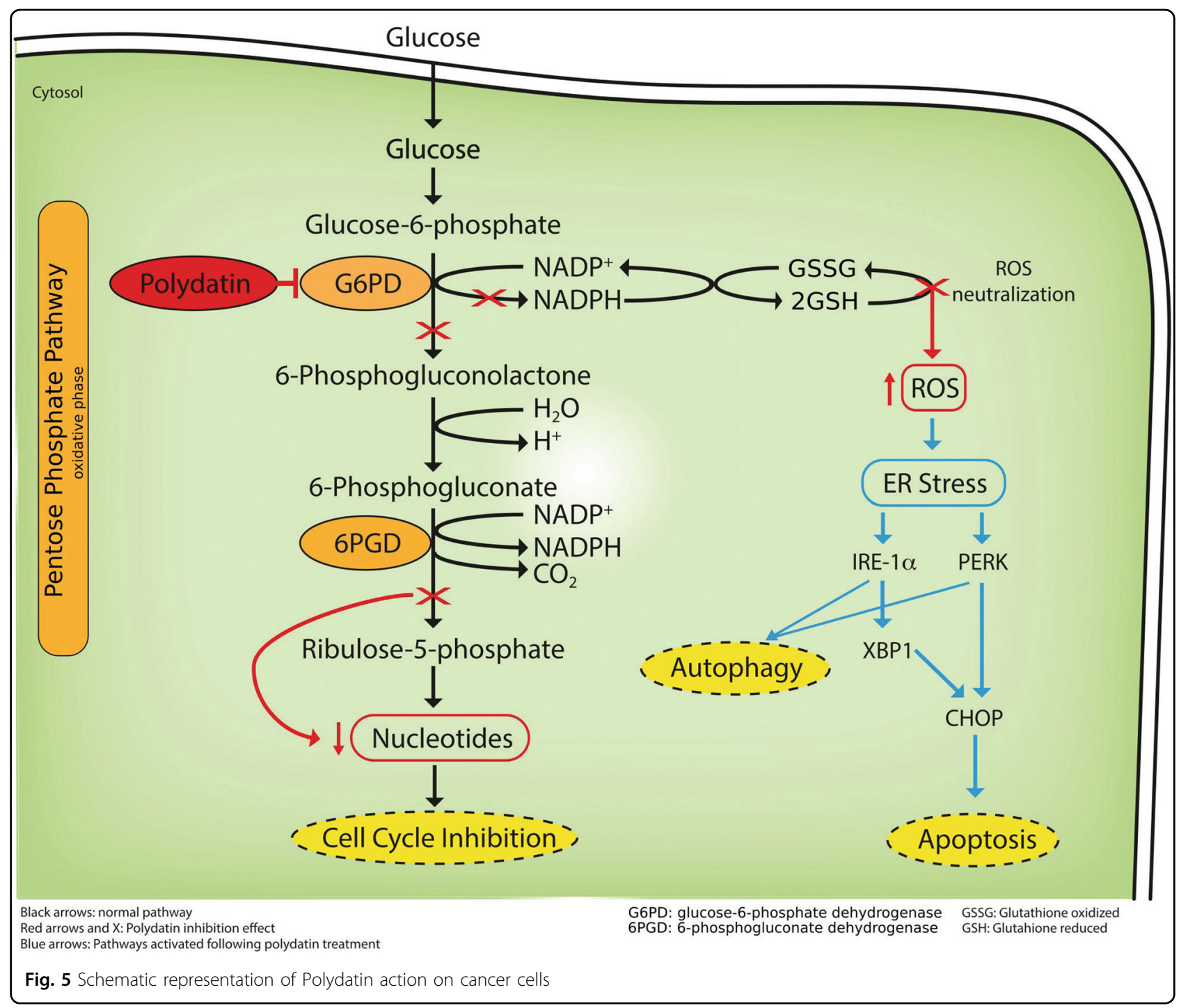

renal toxic effects ${ }^{39,40}$. We showed that the combination of polydatin with either cisplatin or afatinib is strongly synergic in inducing cytotoxicity on cancer cells. These results strongly suggest the use of polydatin in clinical trials in combination with other antitumor agents in integrated anticancer strategies.

In conclusion, we show that polydatin reduces tumor growth and strongly inhibits lymph node metastases in oral cancer in vivo models with no toxicity. This effect has to be correlated with the direct inhibition of G6PD, the limiting enzyme of the PPP. This causes an impairment of NADPH production, causing ROS-mediated ER stress, apoptosis, and invasion inhibition (Fig. 5).

\section{Methods}

Chemicals, cell culture, and in vitro treatment

All chemicals were purchased from Sigma-Aldrich (St. Louis, USA) unless otherwise specified. Trans-polydatin, with a purity grade higher than 99\%, was been supplied by Ghimas spa (Casalecchio, Bologna, Italy). Selective inhibitors of IRE1 $\alpha(4 \mu 8 C)$ and PERK (GSK2606414) were obtained from Tocris Bioscience (Bristol, United Kingdom). Afatinib (BIBW2992) was obtained from Boehringer Ingelheim (Milano, Italy). UMSCC103 (OSCC cell line) used in this study were established at the University of Michigan under a protocol approved by the Institutional Review Board Office in accordance with the university's regulations and described here ${ }^{41}$. MCF7 cells were purchased from ATCC. Cells were cultured in DMEM (Gibco, NY, USA) supplemented with $2 \mathrm{mM}$ glutamine, $100 \mathrm{IU} / \mathrm{mL}$ penicillin, $100 \mu \mathrm{g} / \mathrm{mL}$ streptomycin (Invitrogen, Carlsbad, CA), and 10\% heat-inactivated fetal bovine serum (FBS) (Gibco, NY, USA) at $37^{\circ} \mathrm{C}$ in a humidified atmosphere under $5 \% \mathrm{CO}_{2}$. All cell lines were kept mycoplasma free, checking was performed every 3 months. 


\section{Invasion assay}

The invasion assays were performed using a BD BioCoat Matrigel Invasion Chamber and BD control inserts (Becton-Dickinson, USA). The cells, exposed for $24 \mathrm{~h}$ to DMEM alone and with different concentrations of polydatin, were resuspended in serum-free DMEM and then added onto inserts with uncoated filters (control inserts) or onto inserts with Matrigel coated filters (invasion chambers) at the density of $5 \times 10^{4}$ viable cells/insert. DMEM containing $10 \%$ FBS was used as the chemoattractant. Non-invading cells on the upper side of the membrane, after incubation for $18 \mathrm{~h}$ at $37^{\circ} \mathrm{C}, 5 \% \mathrm{CO}_{2}$, were removed with cotton swabs and the invading cells were fixed using methanol and stained with hematoxylin. Cells that invaded were counted at $\times 200$ magnification. Each assay was performed in triplicate. Cells that invaded the Matrigel insert was compared to the number of cells that migrate in the control insert, calculating as percentage of invasion ${ }^{42}$.

\section{RNA isolation and qRT-PCR}

Total RNA was isolated by RNeasy Mini Kit (Qiagen) according to manufacturer's instructions, RNA was treated with DNase (Promega, Milan, Italy) to exclude DNA contamination and $1 \mu \mathrm{g}$ total RNA reverse-transcribed using VILO SuperScript (Invitrogen, Monza, Italy). Gene expression assays were performed on a StepOne Thermocycler (Applied Biosystems, Monza, Italy) and the amplifications carried out using SYBR Green PCR Master Mix (Applied Biosystems, Monza, Italy). The reaction conditions were as follows: $95^{\circ} \mathrm{C}$ for $15 \mathrm{~min}$, followed by 40 cycles of three steps consisting of denaturation at $94{ }^{\circ} \mathrm{C}$ for $15 \mathrm{~s}$, primer annealing at $60^{\circ} \mathrm{C}$ for $30 \mathrm{~s}$, and primer extension at $72{ }^{\circ} \mathrm{C}$ for $30 \mathrm{~s}$. A melting curve analysis was performed from $70^{\circ} \mathrm{C}$ to $95^{\circ} \mathrm{C}$ in $0.3^{\circ} \mathrm{C}$ intervals. Each sample was performed in triplicate. Glyceraldehyde 3phosphate dehydrogenase (GAPDH) was used to normalize for differences in RNA input. qRT-PCR primer sequences are given in Supplementary Table 2.

\section{G6PD assay and NADP ${ }^{+} /$NADPH quantification}

G6PDH activity was measured by Glucose 6 Phosphate Dehydrogenase Assay Colorimetric Kit (Abcam, Cambridge, UK). UMSCC103 cells untreated or treated with polydatin were homogenized in cold PBS, followed by centrifugation at $8000 \times g$ for $10 \mathrm{~min}$ to remove insoluble materials. G6PDH activity was determined by analysis of G6PDH-dependent oxidation of glucose-6-phospate, which leads to the conversion of a nearly colorless probe to an intensely colored product with an absorbance at 450 $\mathrm{nm}$. All assays were performed at $37^{\circ} \mathrm{C}$. NADP ${ }^{+} / \mathrm{NADPH}$ ratios in cell lines treated with $\mathrm{PD}$ were measured according to the protocol of $\mathrm{NADP}^{+} / \mathrm{NADPH}$ Quantification Kit (MAK038, Sigma). According to the NADPH standards, the concentration of NADPtotal or NADPH can be expressed in pmole per $10^{6}$ cells. The ratio of $\mathrm{NADP}^{+} / \mathrm{NADPH}$ was calculated by ((NADPtotal)(NADPH)/(NADPH)).

\section{Cell viability assay}

Cell viability was measured by the colorimetric 3-(4,5dimethyl-2-thiazolyl)-2,5-diphenyltetrazolium bromide (MTT) assay. Cells were seeded in 96-well plates at a density of $10^{4}$ cells per well, then they were treated with $100 \mu \mathrm{L}$ of $1 \mathrm{mg} / \mathrm{mL}$ MTT (Sigma) in DMEM medium containing $10 \%$ FBS for $4 \mathrm{~h}$ at $37^{\circ} \mathrm{C}$. The medium was then replaced with $200 \mu \mathrm{L}$ of DMSO and shaken for $15 \mathrm{~min}$, then absorbance at $540 \mathrm{~nm}$ was measured using a microplate ELISA reader with DMSO used as the blank. To quantify the synergistic or antagonist effect of the drugs combinations, CompuSyn software was used ${ }^{43}$.

\section{IF staining}

After $24 \mathrm{~h}$ treatment with PD at various concentrations, cells were washed in PBS and fixed with $4 \%$ paraformaldehyde solution and permeabilized with $0.1 \%$ Triton $\mathrm{X} / \mathrm{PBS}$ solution, then was performed a blocking in $1 \%$ BSA for $1 \mathrm{~h}$ at RT. Cells were incubated with anti-pIRE (Abcam, Cambridge, UK) in PBS for $30 \mathrm{~min}$. Secondary antibodies were added after a PBS wash in the same conditions. Cells were incubated in a 1:500 solution of 10 $\mathrm{mg} / \mathrm{mL}$ Hoechst (Invitrogen) in PBS for $10 \mathrm{~min}$ in the dark. To stain ER cells were incubated with 200 nM ERTracker Blue-White DPX in PBS solution for $20 \mathrm{~min}$ at $37^{\circ} \mathrm{C}$. For positive control cells were exposed for $16 \mathrm{~h}$ to $5 \mu \mathrm{g} / \mathrm{mL}$ tunicamycin. Images were collected under a fluorescence microscope (EVOS FL Cell Imaging System, Thermo Scientific, Rockford, USA).

\section{CellROX assay}

Cells were plated on glass bottom 35-mm MatTek dishes and treated with PD for $24 \mathrm{~h}$ or $100 \mu \mathrm{M}$ menadione for $1 \mathrm{~h}$ at $37^{\circ} \mathrm{C}$. A quantity of $50 \mu \mathrm{M} \mathrm{N}$-acetylcysteine was added menadione-treated wells. The cells were then stained with $5 \mu \mathrm{M}$ CellROX green reagent by adding the probe to the complete media and incubating at $37^{\circ} \mathrm{C}$ for $30 \mathrm{~min}$. The cells were then washed with PBS and then imaged on a fluorescence microscope EVOS FL Cell Imaging System (Thermo Scientific, Rockford, USA). Nacetylcysteine treatment inhibited the fluorescent signal induced by menadione, confirming that the signal was specifically produced by ROS increase ${ }^{44,45}$.

\section{FACS analysis}

Apoptosis (Annexin V apoptosis detection kit, BD biosciences), CellROX assay (Thermo Fisher Scientific, USA), were performed according to the manufacturer's instructions. Cells were analyzed with a FACSAria III 
(BD Biosciences, San Jose, CA) or a BD Accuri Cytometer (BD Biosciences, San Jose, CA). Data were analyzed by FlowJo V10 software (FlowJo LLC, USA). For cell cycle analysis, cells were detached from the plates by trypsinization and then fixed with ice-cold $80 \%$ ethanol. The cells were centrifuged and then stained with a solution of 50 $\mu \mathrm{g} / \mathrm{mL}$ propidium iodide and $80 \mu \mathrm{g} / \mathrm{mL}$ RNase A for 60 min at $4{ }^{\circ} \mathrm{C}$ in the dark. DNA content and cell cycle distribution were measured with a FACSARIA III or BD Accuri Cytometer, data were analyzed using Mod-Fit software (Verity Software House, USA).

\section{G6PD overexpression}

p3-G6PD-t1 and negative control pCMV3-untaggedNCV (control) hygroycin-resistant plasmids were purchased from Sino Biological Inc. (Sino Biological, Beijing, China). MCF7 cells were stably transfected with Lipofectamine 3000 (Thermo Fisher Scientific, Waltham, MA USA) according to the manufacturer's instructions. Clones with upregulated expression of G6PD were selected with $100 \mu \mathrm{g} / \mathrm{mL}$ Hygromycin. Clones were screened by western blot.

\section{IRE1 and PERK downregulation}

UMSCC103 cells were transfected with siRNAs targeting IRE1 (SASI_Hs01_00194923, Sigma), PERK (SASI_Hs0100096844, Sigma) or a control siRNA (SIC001, Sigma) using Lipofectamine 3000 (Thermo Fisher Scientific, Waltham, MA, USA) by following the manufacturer's instructions.

\section{Protein extraction and western blotting}

Cells were lysed in 1X RIPA buffer $(150 \mathrm{mM} \mathrm{NaCl}, 1 \%$ NP-40, $0.5 \%$ sodium deoxycholate, $0.1 \%$ SDS, $50 \mathrm{mM}$ Tris- $\mathrm{HCl} \mathrm{pH}$ 7.5) plus $1 \%$ protease inhibitor cocktail, $1 \%$ PMSF $(200 \mathrm{mM})$ and $1 \%$ sodium orthovanadate (Santa Cruz Biotechnology, USA). Lysates were clarified by centrifugation at $8000 \times g$ for $5 \mathrm{~min}$ at $4^{\circ} \mathrm{C}$ and equal amounts of protein were fractionated by SDS-PAGE and subsequently transferred onto nitrocellulose membrane, immunoblots were visualized using Supersignal ${ }^{\circledR}$ West Pico Chemiluminescent substrate (Thermo Scientific, Rockford, USA). Proteins were detected with anti-Glucose 6 Phosphate Dehydrogenase (Novus Biologicals, USA); anti-PERK (phospho T981) (\#1055, Elabscience, Huston, USA); anti-PERK (\#3667, Elabscience, Huston, USA); anti-IRE1 (phospho S724) (ab48187, Abcam, Cambridge, UK); anti-IRE1 (ab ab37073, Abcam, Cambridge, UK); Anti-eIF2 $\alpha$ (phosphor S51) (9721, Cell Signaling, USA); anti- $\alpha$-Tubulin Antibody (\#2144 Cell Signalling Technology, UK); Anti-Calreticulin (ab2907, Abcam, Cambridge, UK); Anti-Calnexin (ab22595, Abcam, Cambridge, UK); Anti-BiP (BD610978, BD Biosciences, San Jose, CA); Anti-ATF4 (sc-200, Santa Cruz, Dallas, USA); Anti-Chop (sc7351, Santa Cruz, Dallas, USA); Anti-XBP1 (ab198999, Abcam, Cambridge, UK) and Anti-GAPDH (ab9485, Abcam, Cambridge, UK) were used for assessing loading.

\section{Mass spectrometry}

Samples ( $\sim 50 \mu$ g protein) were reduced, alkylated, and prepared as previously described ${ }^{46}$. Samples $(3 \mu \mathrm{L}, \sim 5 \mu \mathrm{g}$ protein digest) were directly injected by autosampler (Eksigent nanoLC $425 \mathrm{LC}$ system) at $5 \mu \mathrm{L} / \mathrm{min}$ onto a YMC Triart- $\mathrm{C}_{18}$ column $(15 \mathrm{~cm}, 3 \mu \mathrm{m}, 300 \mu \mathrm{m}$ i.d.) using gradient elution (2-40\% mobile phase B, followed by wash at $80 \% \mathrm{~B}$ and re-equilibration) over either 73 (120 min run time) min (for spectral library construction using data/ information dependent acquisition DDA/ IDA) or $43 \mathrm{~min}$ (60 min run time) for SWATH/DIA (data independent acquisition) analysis. Mobile phases consisted of A: water containing $0.1 \%(\mathrm{v} / \mathrm{v})$ formic acid; B: acetonitrile containing $0.1 \%(\mathrm{v} / \mathrm{v})$ formic acid. The LC system was hyphenated to a Sciex TripleTof $5600+$ mass spectrometer fitted with a Duospray source and $50 \mu \mathrm{m}$ electrode suitable for microflow proteomic analysis. The IDA method was run with parameters of: CUR 25; GS1 15; GS2 0; ISVF 5500; TEM 0. TOFMS mass range of $400-1250 \mathrm{~m} / \mathrm{z}$; accumulation time of $250 \mathrm{~ms}$ with product ion scans on the top 30 ions before switching (dynamic exclusion for $20 \mathrm{~s}$ ) with rolling collision energy selected. Product ion accumulation time was set to $50 \mathrm{~ms}$ giving a cycle time of $1.8 \mathrm{~s}$. The SWATH method was run with the same source parameters as the IDA with a $50 \mathrm{~ms}$ TOFMS scan followed by 100 variable SWATH windows (optimized on an IDA datafile of the same samples) of $25 \mathrm{~ms}$ between 100 and $1500 \mathrm{~m} / \mathrm{z}$ giving a cycle time of $2.6 \mathrm{~s}$.

A spectral library for SWATH data extraction was constructed using the output from ProteinPilot 5.0 (Sciex) searching against the Swissprot Human database (January 2015) with the addition of iRT peptides to the. fasta file, combining eight IDA runs (pooled samples) and filtered and aligned to spike in iRT peptides (Biognosys, Switzerland) using PeakView 2.1 (Sciex). SWATH data extraction, quantitation, and fold change analysis were carried out using Sciex's OneOmics cloud processing software suite incorporating processing methodology from ref. ${ }^{47}$. Proteins were considered as differentially expressed if they had quantitative data on more than a single peptide, with a OneOmics confidence threshold of $70 \%$.

\section{In vivo experiments}

Twenty Foxn1nu nude male mice (6-8 weeks old) were purchased from the Harlan, Italy (Sanpietro al Natisone). The mice were maintained under pathogen-free conditions with food and water ad libitum, on 12/12 h day/night cycle, a temperature of $20 \pm 2{ }^{\circ} \mathrm{C}$, ten mice per cage randomized according to weight and tumor burden and divided in two treatment groups respectively; control 
vehicle (ethanol 100\%) and polydatin $100 \mathrm{mg} / \mathrm{kg}$ i.p. UMSCC103 cells were trypsinized, centrifuged, and then resuspended in serum-free medium. For implantation, tumor cells were orthotopically inoculated into the right lateral portion of the tongue of mice $\left(1 \times 10^{6}\right.$ cells per mouse) using a syringe with a 30 gauge disposable needle (BD Biosciences). Animals were anesthetized with intraperitoneal injections of zolazepam, atropine and xilazine. From 10 days post injection, mice were treated three times a week with either control vehicle or polydatin 100 $\mathrm{mg} / \mathrm{kg}$ via i.p. Tumor growth was assessed every 3 days, and cervical lymph node metastasis were evaluated with Ultrasound System Vevo 2100 (Visualsonics, Canada) at 10 and 20 days post cell injection. For each group the area of the cervical lymph node was assessed with the Vevo 2100 commercial software. At the end of the experiment (30 days of treatments), mice were killed by $\mathrm{CO}_{2}$ inhalation, dissected, and the tongue and cervical lymph nodes weighed and photographed. The experimental protocols were in compliance with the European Community Council directive (86/609/EEC).

\section{Acknowledgements}

We would like to thank Prof. Claudio Napoli for critical review of the manuscript and for precious advice he gave us, Prof. Silvana Papagerakis who provided UMSCC103 cell line, Prof. Francesco Fazi who provided antibodies to analyze UPR pathway. This research is supported by the following grants: Second University of Naples "Avvio alla Ricerca Scientifica 2015" (F. Paino, V.D., and V.T.); Italian Ministry of Education Research and University PON03PE_000607 2013/2017 (G.P.); grant entitled “Laboratori Pubblici Progetto Hauteville" from Campania Regional Government (M.C).

\section{Author details \\ 'Department of Experimental Medicine, University of Campania "Luigi Vanvitelli", 80138 Naples, Italy. ${ }^{2}$ Oncologia Medica ed Ematologia, Dipartimento Medico-Chirurgico di Internistica Clinica e Sperimentale "F. Magrassi e A. Lanzara", University of Campania "Luigi Vanvitelli", 80138 Naples, Italy. ${ }^{3}$ The John van Geest Cancer Research Centre, School of Science and Technology, Nottingham Trent University, Clifton Lane, NG1 4FQ Nottingham, UK. ${ }^{4}$ Department of Biochemistry, Biophysics and General Pathology, University of Campania "Luigi Vanvitelli", 80138 Naples, Italy. ${ }^{5}$ Department of Research, Pathology Unit, Istituto Nazionale Tumori- IRCCS- Fondazione Pascale, 80131 Naples, Italy. ${ }^{6}$ SSD Sperimentazione Animale, Istituto Nazionale Tumori- IRCCS- Fondazione Pascale, 80131 Naples, Italy}

\section{Author contributions}

L.M., F. Paino, and F. Papaccio performed all in vitro experiments with the help of D.L., M.L.N., A.L.; C.A. and A.B. performed in vivo studies; G.A. performed histopathology analysis of tumor tissues collected from mice; D.B. and C.C. performed mass spectrometry and related data analysis; P.S. collected and assembled data; T.R., G.P. interpreted data and edited the manuscript; V.D., M. C., and V.T. designed the study, assembled, and interpreted data, wrote the manuscript.

\section{Conflict of interest}

The authors declare that they have no conflict of interest.

\section{Publisher's note}

Springer Nature remains neutral with regard to jurisdictional claims in published maps and institutional affiliations.
Supplementary Information accompanies this paper at (https://doi.org/ 10.1038/s41419-018-0635-5).

Received: 23 March 2018 Revised: 10 April 2018 Accepted: 17 April 2018 Published online: 14 May 2018

\section{References}

1. Jiang, P., Du, W. \& Wu, M. Regulation of the pentose phosphate pathway in cancer. Protein Cell 5, 592-602, (2014).

2. Horecker, B. L. The pentose phosphate pathway. J. Biol. Chem. 277, 47965-47971, (2002).

3. Kruger, N. J. \& von Schaewen, A. The oxidative pentose phosphate pathway: structure and organisation. Curr. Opin. Plant Biol. 6, 236-246 (2003).

4. Patra, K. C., \& Hay, N. The pentose phosphate pathway and cancer. Trends Biochem. Sci. 39, 347-354 (2014).

5. Riganti, C., Gazzano, E., Polimeni, M., Aldieri, E., \& Ghigo, D. The pentose phosphate pathway: an antioxidant defense and a crossroad in tumor cell fate. Free Radic. Biol. Med. 53, 421-436 (2012).

6. Du, W. et al. TAp73 enhances the pentose phosphate pathway and supports cell proliferation. Nat. Cell Biol. 15, 991-1000, (2013).

7. Lin, R. et al. 6-Phosphogluconate dehydrogenase links oxidative PPP, lipogenesis and tumour growth by inhibiting LKB1-AMPK signalling. Nat. Cell Biol. 17, 1484-1496, (2015)

8. Compagno, M. et al. Mutations of multiple genes cause deregulation of NFkappaB in diffuse large B-cell lymphoma. Nature 459, 717-721, (2009).

9. Jiang, P., Du, W. \& Yang, X. A critical role of glucose-6-phosphate dehydrogenase in TAp73-mediated cell proliferation. Cell Cycle 12, 3720-3726, (2013).

10. Quade, B. J. et al. Molecular pathogenesis of uterine smooth muscle tumors from transcriptional profiling. Genes Chromosomes Cancer 40, 97-108, (2004).

11. Rosenwald, A. et al. The use of molecular profiling to predict survival after chemotherapy for diffuse large-B-cell lymphoma. N. Engl. J. Med 346, 1937-1947, (2002).

12. Stearman, R. S. et al. Analysis of orthologous gene expression between human pulmonary adenocarcinoma and a carcinogen-induced murine model. Am. J. Pathol. 167, 1763-1775, (2005).

13. Debeb, B. G. et al. Histone deacetylase inhibitor-induced cancer stem cells exhibit high pentose phosphate pathway metabolism. Oncotarget 7, 28329-28339, (2016)

14. Gyorffy, B. et al. An online survival analysis tool to rapidly assess the effect of 22,277 genes on breast cancer prognosis using microarray data of 1,809 patients. Breast Cancer Res. Treat. 123, 725-731, (2010).

15. Ringner, M., Fredlund, E., Hakkinen, J., Borg, A. \& Staaf, J. GOBO: gene expression-based outcome for breast cancer online. PLOS ONE 6, e17911, (2011).

16. Catanzaro, D. et al. Inhibition of glucose-6-phosphate dehydrogenase sensitizes cisplatin-resistant cells to death. Oncotarget 6, 30102-30114, (2015).

17. Jones, N. P. \& Schulze, A. Targeting cancer metabolism-aiming at a tumour's sweet-spot. Drug Discov. Today 17, 232-241, (2012).

18. Di Monaco, M. et al. Role of glucose-6-phosphate dehydrogenase inhibition in the antiproliferative effects of dehydroepiandrosterone on human breast cancer cells. Br. J. Cancer 75, 589-592 (1997).

19. Liu, H. et al. Reactive oxygen species-mediated endoplasmic reticulum stress and mitochondrial dysfunction contribute to polydatin-induced apoptosis in human nasopharyngeal carcinoma CNE cells. J. Cell Biochem. 112, 3695-3703, (2011).

20. Dandawate, P. R., Subramaniam, D., Jensen, R. A., \& Anant, S. Targeting cancer stem cells and signaling pathways by phytochemicals: novel approach for breast cancer therapy. Semin. Cancer Biol. 40-41, 192-208 (2016).

21. Kong, Y. et al. Pterostilbene induces apoptosis and cell cycle arrest in diffuse large B-cell lymphoma cells. Sci. Rep. 6, 37417, (2016).

22. $\mathrm{Cai}, \mathrm{H}$. et al. Cancer chemoprevention: evidence of a nonlinear dose response for the protective effects of resveratrol in humans and mice. Sci. Transl. Med. 7, 298ra117, (2015).

23. Gregory, M. A. et al. ATM/G6PD-driven redox metabolism promotes FLT3 inhibitor resistance in acute myeloid leukemia. Proc. Natl Acad. Sci. USA 113 E6669-E6678, (2016).

24. Pavlova, N. N. \& Thompson, C. B. The emerging hallmarks of cancer metabolism. Cell Metab. 23, 27-47, (2016). 
25. Cairns, R. A., Harris, I. S. \& Mak, T. W. Regulation of cancer cell metabolism. Nat. Rev. Cancer 11, 85-95, (2011).

26. Vander Heiden, M. G., Cantley, L. C. \& Thompson, C. B. Understanding the Warburg effect: the metabolic requirements of cell proliferation. Science $\mathbf{3 2 4}$ 1029-1033, (2009).

27. Jiang, Q., Wong, J. \& Ames, B. N. Gamma-tocopherol induces apoptosis in androgen-responsive LNCaP prostate cancer cells via caspase-dependent and independent mechanisms. Ann. N. Y. Acad. Sci. 1031, 399-400, (2004).

28. Rohrig, F., \& Schulze, A. The multifaceted roles of fatty acid synthesis in cancer. Nat. Rev. Cancer 16, 732-749 (2016).

29. Tian, W. N. et al. Importance of glucose-6-phosphate dehydrogenase activity for cell growth. J. Biol. Chem. 273, 10609-10617 (1998).

30. Zhang, Y. et al. Polydatin inhibits growth of lung cancer cells by inducing apoptosis and causing cell cycle arrest. Oncol. Lett. 7, 295-301, (2014).

31. Anglana, M., Apiou, F., Bensimon, A. \& Debatisse, M. Dynamics of DNA replication in mammalian somatic cells: nucleotide pool modulates origin choice and interorigin spacing. Cell 114, 385-394 (2003).

32. Piskounova, E. et al. Oxidative stress inhibits distant metastasis by human melanoma cells. Nature 527, 186-191, (2015).

33. Richardson, A. D., Yang, C., Osterman, A. \& Smith, J. W. Central carbon metabolism in the progression of mammary carcinoma. Breast Cancer Res. Treat. 110, 297-307, (2008).

34. Chen, E. I. et al. Adaptation of energy metabolism in breast cancer brain metastases. Cancer Res. 67, 1472-1486, (2007).

35. Du, J. et al. Lipid-lowering effects of polydatin from Polygonum cuspidatum in hyperlipidemic hamsters. Phytomedicine 16, 652-658, (2009).

36. Xing, W. W. et al. Effects of polydatin from Polygonum cuspidatum on lipid profile in hyperlipidemic rabbits. Biomed. Pharmacother. 63, 457-462, (2009).

37. Wang, H. L. et al. Comparative studies of polydatin and resveratrol on mutual transformation and antioxidative effect in vivo. Phytomedicine 22, 553-559 (2015).
38. Du, Q. H., Peng, C., \& Zhang, H. Polydatin: a review of pharmacology and pharmacokinetics. Pharm. Biol. 51, 1347-1354 (2013).

39. Cremon, C. et al. Randomised clinical trial: the analgesic properties of dietary supplementation with palmitoylethanolamide and polydatin in irritable bowel syndrome. Aliment. Pharmacol. Ther. 45, 909-922, (2017).

40. Indraccolo, U., Indraccolo, S. R. \& Mignini, F. Micronized palmitoylethanolamide/trans-polydatin treatment of endometriosis-related pain: a meta-analysis. Ann. Ist. Super. Sanita 53, 125-134, (2017).

41. Desiderio, $V$. et al. Increased fucosylation has a pivotal role in invasive and metastatic properties of head and neck cancer stem cells. Oncotarget $\mathbf{6 , 7 1 - 8 4 ,}$ (2015).

42. Tirino, V. et al. TGF-beta1 exposure induces epithelial to mesenchymal transition both in CSCS and non-CSCS of the A549 cell line, leading to an increase of migration ability in the CD133+ A549 cell fraction. Cell Death Dis. 4, e620 (2013).

43. Chou, T. C. Drug combination studies and their synergy quantification using the Chou-Talalay method. Cancer Res. 70, 440-446, (2010).

44. Bruzzese, F. et al. Panobinostat synergizes with zoledronic acid in prostate cancer and multiple myeloma models by increasing ROS and modulating mevalonate and p38-MAPK pathways. Cell Death Dis. 4, e878 (2013).

45. Caraglia, M. et al. Oxidative stress and ERK1/2 phosphorylation as predictors of outcome in hepatocellular carcinoma patients treated with sorafenib plus octreotide LAR. Cell Death Dis. 2, e150 (2011).

46. Vyas, F. S. et al. A1 adenosine receptor-induced phosphorylation and modulation of transglutaminase 2 activity in $\mathrm{H} 9 \mathrm{c} 2$ cells: a role in cell survival. Biochem. Pharmacol. 107, 41-58 (2016).

47. Lambert, J. P. et al. Mapping differential interactomes by affinity purification coupled with data-independent mass spectrometry acquisition. Nat. Methods 10, 1239-1245, (2013). 